\title{
APOE $\varepsilon 4$ moderates abnormal CSF-abeta-42 levels, while neurocognitive impairment is associated with abnormal CSF tau levels in HIV+ individuals - a cross-sectional observational study
}

Lucette A Cysique ${ }^{1,2,3,4^{*}}$, Timothy Hewitt ${ }^{4,5}$, Juliana Croitoru-Lamoury ${ }^{1,4}$, Kevin Taddei $^{6,7}$, Ralph N Martins ${ }^{6,7,8}$, Constance SN Chew ${ }^{9}$, Nicholas NWS Davies ${ }^{10}$, Patricia Price ${ }^{9}$ and Bruce J Brew ${ }^{1,3,4}$

\begin{abstract}
Background: Cerebrospinal fluid (CSF) biomarkers Aß1-42, t-tau and p-tau have a characteristic pattern in Alzheimer's Disease (AD). Their roles in HIV-associated neurocognitive disorder (HAND) remains unclear.

Methods: Adults with chronic treated HIV disease were recruited ( $n=43$, aged $56.7 \pm 7.9 ; 32 \%$ aged $60+$; median HIV duration 20 years, $>95 \%$ plasma and CSF HIV RNA $<50 \mathrm{cp} / \mathrm{mL}$, on CART for a median 24 months). All underwent standard neuropsychological testing (61\% had HAND), APOE genotyping (30.9\% carried APOE $\varepsilon 4$ and $7.1 \%$ were $\varepsilon 4$ homozygotes) and a lumbar puncture. Concentrations of A 1 1-42, t-tau and p-tau were assessed in the CSF using commercial ELISAs. Current neurocognitive status was defined using the continuous Global Deficit Score, which grades impairment in clinically relevant categories. History of HAND was recorded. Univariate correlations informed multivariate models, which were corrected for nadir CD4-T cell counts and HIV duration.

Results: Carriage of APOE $\varepsilon 4$ predicted markedly lower levels of CSF Aß1-42 in univariate $(r=-.50 ; p=.001)$ and multivariate analyses $\left(R^{2}=.25 ; p<.0003\right)$. Greater levels of neurocognitive impairment were associated with higher CSF levels of $\mathrm{p}$-tau in univariate analyses $(r=.32 ; p=.03)$ and multivariate analyses $\left(R^{2}=.10 ; p=.03\right)$. AD risk prediction cut-offs incorporating all three CSF biomarkers suggested that $12.5 \%$ of participants had a high risk for AD. Having a CSF-AD like profile was more frequent in those with current $(p=.05)$ and past HIV-associated dementia $(p=.03)$.

Conclusions: Similarly to larger studies, APOE $\varepsilon 4$ genotype was not directly associated with HAND, but moderated CSF levels of Aß1-42 in a minority of participants. In the majority of participants, increased CSF p-tau levels were associated with current neurocognitive impairment. Combined CSF biomarker risk for AD in the current HIV+ sample is more than 10 times greater than in the Australian population of the same age. Larger prospective studies are warranted.
\end{abstract}

Keywords: HIV/AIDS, CSF, APOE, Aß1-42, t-tau, p-tau, HIV-associated neurocognitive disorders

\section{Background}

The cerebrospinal fluid (CSF) biomarkers A $\beta 1-42$, $t$-tau and $\mathrm{p}$-tau have a characteristic pattern in Alzheimer's Disease (AD) (AD profile: low levels of $A \beta 1-42$ and high levels of $t$-and $p$-tau $[1,2]$ ) and may be associated with the cognitive changes seen in people with HIV-associated

\footnotetext{
* Correspondence: Icysique@unsw.edu.au

'University of New South Wales, St. Vincent's Hospital Clinical School, Sydney, Australia

${ }^{2}$ Neuroscience Research Australia, Sydney, Australia

Full list of author information is available at the end of the article
}

neurocognitive disorder (HAND). Links between AD and HAND are becoming increasingly relevant as AD is generally associated with age, and HIV+ individuals now live longer on effective combined antiretroviral therapy (cART). Also, there is some preliminary evidence for accelerated aging of the brain in middle-aged HIV+ individuals [3]. Three studies have investigated a potential link between these two disease entities. Brew and colleagues found an AD-like pattern of CSF A $\beta 1-42$, t-tau and p-tau concentrations in mild to moderate HAND $(\mathrm{N}=87 ; 46$ AIDS-dementia complex (ADC) stage 1; 41 ADC stage 2;

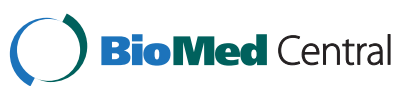

(C) 2015 Cysique et al.; licensee BioMed Central. This is an Open Access article distributed under the terms of the Creative Commons Attribution License (http://creativecommons.org/licenses/by/4.0), which permits unrestricted use, distribution, and reproduction in any medium, provided the original work is properly credited. The Creative Commons Public Domain Dedication waiver (http://creativecommons.org/publicdomain/zero/1.0/) applies to the data made available in this article, unless otherwise stated. 
age: $\mu=42$; $\mathrm{SD}=11$ ) consistent with that seen in $\mathrm{AD}$ [4]. A study in HIV+ individuals with HAND $(\mathrm{N}=21$; 3 ADC stage 1; 18 ADC stages 2-4; age: $\mu=38$; $S D=18$;) reported low levels of $A \beta 1-42$ but normal levels of $t$-tau and $p$-tau [5]. Another study ( $\mathrm{N}=49 ; 30 \mathrm{MCD} ; 11 \mathrm{HAD} ; 8$ unknown; age: $\mu=48$; $\mathrm{SD}=8$ ) reported low levels of $\mathrm{A} \beta 1-42$ and $\mathrm{t}$-tau and p-tau [6]. These inconsistent results may reflect heterogeneity in the severity of HAND, the efficacy of cART, HIV disease duration and/or patient age.

Importantly, no study included APOE (Apolipoprotein E) genotyping, which may affect AD independently, or in association with, CSF biomarkers depending on the age of the subject [7]. The $\varepsilon 4$ allele of APOE, an apolipoprotein thought to be partially responsible for amyloid clearance in the CNS, is one of the greatest known risk factors for late-onset sporadic AD [8,9]. Large cohort studies have provided conflicting results regarding the role of APOE in HIV+ persons with HAND. A study based on the Hawaii Aging with HIV Cohort [10] $(\mathrm{N}=182)$ associated APOE \&4 carriage with HAND, but only in older participants. The largest study conducted in an ethnically diverse cohort [11] (Wilford Hall Medical Center, WHMC; 1,267 HIV-seropositive adults and 1,132 ethnically similar HIV-seronegative controls) found an association between APOE $\varepsilon 4 / \varepsilon 4$ genotype and acceleration of HIV disease, but not with HAD. However the authors only assessed HAD rather than the complete HAND spectrum. Moreover, it is not clear how HAD was defined and assessed. More recently the CNS HIV Antiretroviral Therapy Effects Research (CHARTER) study [12] of $466 \mathrm{HIV+}$ participants (mean age $=44$ ) who received a comprehensive HAND assessment showed no association between APOE $\varepsilon 4$ carriage and HAND. They similarly found no association when they restricted their analyses to those with moderate HAND (Mild Neurocognitive Disorder (MND) and HAD). Age did not influence their results, but their sample included only $3.7 \%$ of persons aged $60+$, compared to $25 \%$ in the Hawaii Aging with HIV Cohort [10]. The authors conclude that within the age-group they investigated APOE was not associated with HAND, confirming other smaller studies in same-age or younger samples [13-15], but stated that their results does not "preclude emergence of an association between APOE status and HAND as this population ages", so further studies are needed.

The current study included 43 chronic HIV+ adults aged $56.7 \pm 7.9(32 \%$ aged $60+)$ years on long-term cART with no detectable HIV RNA in their plasma or CSF. The aims were: 1 . Investigate whether the biomarkers $A \beta 1-42$, t-tau and $p$-tau follow a similar pattern to that found in $\mathrm{AD}$ and assess the prevalence of CSF-AD like profile. 2. Investigate the relationship between APOE genotype, CSF biomarker levels and severity of HAND in these individuals. We hypothesized that
HIV+ adults with HAND would be more likely to have a CSF AD-like profile than those who did not have HAND. Our second aim was exploratory, as APOE genotypes have not ben correlated CSF markers in chronic HIV infection.

\section{Methods \\ Study participants}

Participants were recruited through the HIV and Neurology Clinics at St Vincent's Hospital, Sydney, Australia. Eligibility criteria included: age $\geq 45$ years, stable cART $\geq 6$ months, nadir CD4 T-cells $\leq 350 / \mathrm{ul}$ and HIV duration $\geq 5$ years. Exclusion criteria included a history of non-HIV related neurological disorders or uncontrolled axis I psychiatric disorders, history of psychotic disorder, substance/alcohol use disorders (DSM-IV) within 12 months of enrolment, history of loss of consciousness $>30$ minutes, and non-proficiency in English (published protocol details $[3,16])$. St. Vincent's Hospital, The University of New South Wales, and the University of Western Australia Human Research Ethics Committees approved protocols. All participants provided written informed consent.

\section{Current impairment status}

Detailed testing procedures have been published Lane et al. [16] and Cysique et al. [17]. Briefly, HIV+ participants underwent a standard neuropsychological evaluation assessing seven cognitive domains. Impairment status was determined using local normative standards (z-scores) developed in a demographically comparable HIV- sample recruited as part of the HIV and Brain Aging research program at the University of New South Wales (PI, LAC). Details on methods and the sample characteristics used to develop local norms have been published [17]. The standard Global Deficit Score (GDS) method [18,19] was used to classify impairment. As per convention [18,19], GDS $\geq 0.5$ was used to define a clinically relevant level of impairment yielding a discrete outcome (impaired or unimpaired) or a continuous outcome. A higher GDS indicates greater impairment. The standard GDS cut-off of $\geq 0.5$ is widely used to assess HIV-related brain injury and meets the Frascatti criteria for HAND [20]. For each participant, we determined the HAND classification (Asymptomatic Neurocognitive Impairment: ANI; Mild Neurocognitive disorder: MND; or HAD) as follows: [20]. GDS $\geq 0.5$ \& no Independence in Activities of Daily Living (IADL) [21] decline $=$ ANI; GDS $\geq 0.5$ \& mild $/$ moderate IADL decline = MND; GDS $\geq 1.5$ \& severe IADL decline $=$ HAD. IADL information was obtained from a standard IADL scale, the Patients Assessment of Own Functioning Inventory [22] and any clinical evidence of IADL decline (medical record; nurse information). 


\section{Past HAND status}

History of HAND was based on standard neurological and neuropsychological examinations, MRI/MRS scans and an extended panel of plasma and CSF biomarkers, as described previously [23]. LC consulted the medical records and recorded HAND diagnoses reported using the AIDS Dementia Complex nomenclature [24]. This was adapted to the HAND 2007 criteria [25] by LC (see Additional file 1) and reviewed by BJB (HIV Neurologist) to reach clinically relevant diagnoses of MND and HAD [25]. Past HAND status was also analysed as a dichomotous variable.

\section{Specimen collection and quantitation of biomarkers in cerebrospinal fluid}

CSF was stored at $-70^{\circ} \mathrm{C}$ until assayed. CSF A $\beta 1-42$ concentrations were determined using a sandwich enzyme-linked immunoassay (ELISA) with a limit of detection of $15 \mathrm{pg} /$

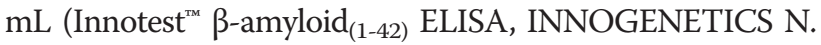
$\mathrm{V}$, Ghent, Belgium). CSF t-tau and p-tau concentrations were determined using a sandwich ELISA with limits of detection of 87 and $15 \mathrm{pg} / \mathrm{mL}$ (Innotest ${ }^{\mathrm{TM}}$ hTAU Antigen assay and Innotest $^{\mathrm{Tm}}$ PHOSPHO-TAU ${ }_{(181 \mathrm{P})}$ ELISA, INNOGENETICS N.V). As within-laboratory variation has been reported [26], we tested our procedure in a eight HIV-controls, comprising three cognitively healthy adults and five adults with $\mathrm{AD}$ recruited from the Memory Clinics of the Sir James McCusker Alzheimer's Disease Research Unit (Western Australia) and the Neurology AD clinics at St. Vincent's Hospital. AD diagnoses were based on the NINCS-ADRA [27] and DSM-IV [28] criteria. CSF samples were examined blind to the AD \& HIV status and vice versa. CSF markers concentrations are presented in Figure 1. Laboratory analyses were reliable across duplicates $(p<.0001)$. To examine the sensitivity and specificity of our assay procedure, we used published cut-offs [29] which display sensitivity (95\%) and specificity $(\geq 81 \%)$ for $\mathrm{AD}$ and incipient $\mathrm{AD}$ [30]: CSF-AD profiles were 1). t-tau >350 \& A $\beta 1-42<530$ pg/ml; 2). p-tau $>60 \& A \beta 1-$ $42<530 \mathrm{pg} / \mathrm{ml}$; 3). t-tau $>350 \mathrm{pg} / \mathrm{ml} \& \mathrm{~A} \beta 1-42 / \mathrm{p}$-tau $<6.5$.
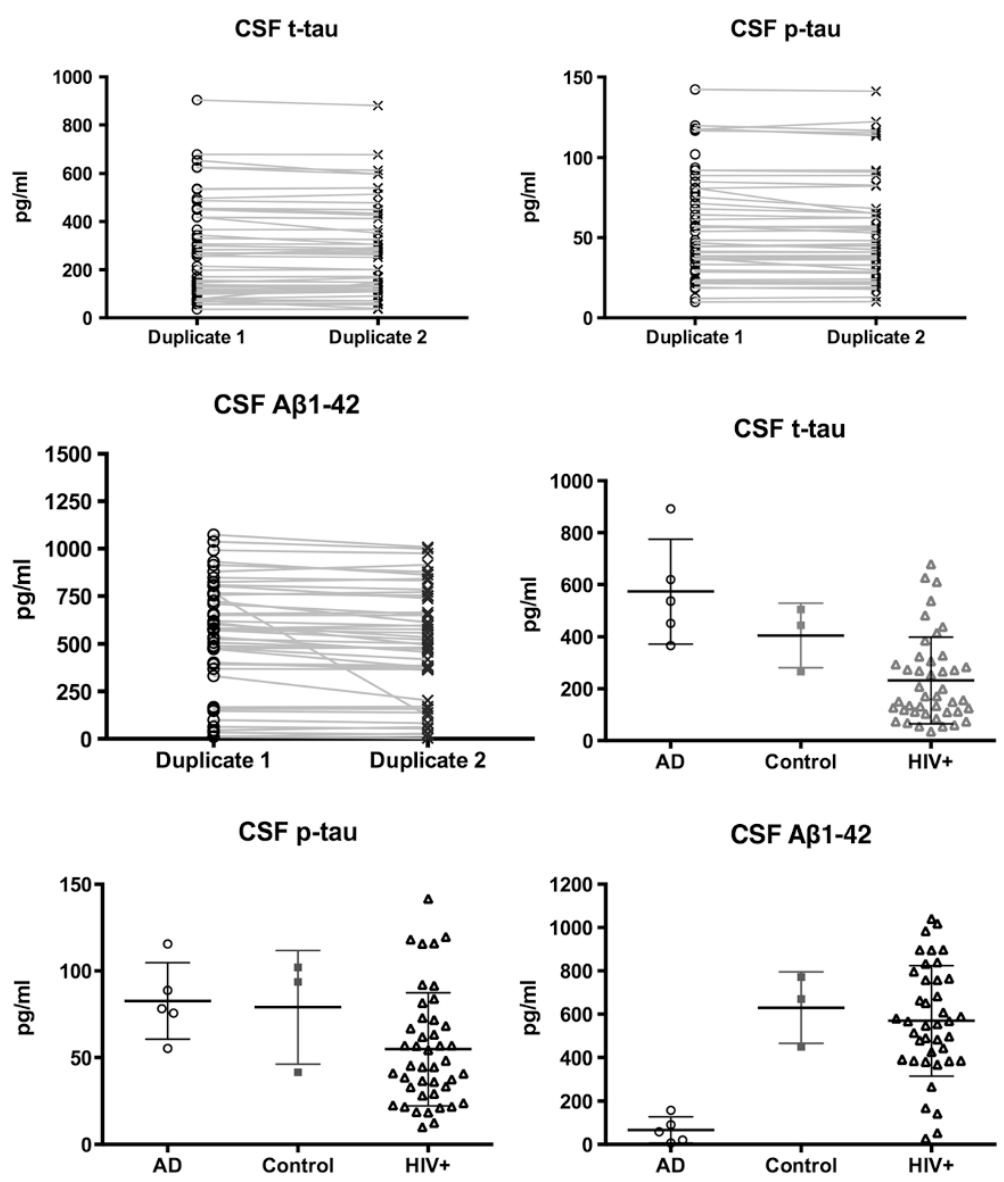

Figure 1 Raw duplicate and average data for the CSF biomarkers. To assess the reliability of all the CSF biomarker concentrations, we performed correlations between assays duplicate for $t$-tau, $p$-tau $(r=.99 ; p<.0001)$ and A $\mathrm{A} 1-42(r=.95 ; p<.0001)$. They were highly reliable. To assess the within group duplicate reliability we conducted a t-test between duplicate 1 and 2 within HIV, AD and HIV- group and all results were non-significant differences (t-tau; p-tau: $p>$.90; A $1-42 p>.50$ ). 
At least one CSF-AD profile was found in all $\mathrm{AD}$ patients and none in the HIV- controls (see Figure 2). CSF A $\beta 1-42$ was missing in three cases because there was insufficient CSF, so cut-offss were based on 40 cases.

\section{APOE genotyping}

DNA was extracted from saliva or blood leukocytes using QIAamp DNA mini Kits (QIAGEN; Valencia, CA) and stored at $-80^{\circ} \mathrm{C}$. Two SNPs in the APOE gene (rs429358, rs7412) defining the $A P O E \varepsilon 3, \varepsilon 4$ and $\varepsilon 2$ variants were genotyped using TaqMan assays according to manufacturer's instructions. Median (range) genotyping success rates were $99 \%(98 \%-100 \%)$.

\section{Data analyses}

Categorical data were assessed with the Fisher's Exact Test. Concentrations of $\mathrm{t}$-tau, $\mathrm{p}$-tau were $\log _{10}$ transformed to approximate normal distributions. Univariate analyses (Pearson and/or point biserial correlations) evaluated associations between the CSF biomarkers and AD risk factors [31] (APOE genotypes: homozygotes $(\varepsilon 4 / \varepsilon 4)$ vs. heterozygotes $(\varepsilon 4 / \varepsilon 2$ or $\varepsilon 4 / \varepsilon 3)$ vs no $\varepsilon 4$, and age), HIV-related parameters [32] (nadir CD4 T-cell count and HIV duration in years) and neurocognition (current GDS and past HAND status).

Multivariate analyses comprised stepwise regression models with CSF p-tau (t-tau was excluded as it correlated with $\mathrm{p}$-tau) and A $\beta 1-42$ separately as outcomes. For the $A \beta 1-42$, predictors were included in the order defined above. For p-tau, the continuous GDS was entered first (based on univariate analyses), followed by past HAND status, age, HIV disease markers and APOE status. The model was re-run removing a priori APOE and past HAND status. We used a forward model selection with the Akaike Information Criterion corrected
(AICc), as it is less reliant on p-value than other type of stepwise selection methods. The model with the lowest AICc yields the best compromise between goodness to fit and model complexity [33]. Statistical analyses were conducted using JMP 10 (SAS Inc).

\section{Results}

Demographic and clinical characteristics of the HIV+ group are presented in Table 1, whilst characteristics of the reference samples are presented in Additional file 2. HIV+ participants were characterized by historic immune compromise, which had improved substantially on cART. Only 2 cases had detectable HIV RNA in plasma and one other in the CSF. These are considered to be "detection blips" as participants were clinically stable on cART and negative HIV RNA assessments had been recorded within the previous 12 months (data not shown). Most participants were well-educated white men. The AD and control sample were older than the HIV+ sample by design, but comparable for other demographics. They were included to demonstrate reliability of CSF assays and CSF-AD like profiling.

When the cut-offs distinguishing AD in HIV- and AD cases (described in methods) were applied, 5\% (2/40) HIV+ participants had profile $1,7.5 \%(3 / 40)$ had profile 2 and $7.5 \%$ (3/40) had profile 3. One participant met the AD-CSF profile on all 3 cut-offs. In total, 12.5\% (5/40) had at least one CSF-AD like profile (Figure 2).

A diagnosis of HAND (versus GDS $\geq 0.5$ ) did not associate with having a CSF AD- like profile $(12.5 \%$ with or without HAND). However, those with HAD (40\%) were more likely to have a CSF AD-like profile than those with ANI $(0 \% ; p=.05)$. They were also marginally more likely to have a CSD AD-like profile compared to with MND (16.7\%), but this was not statistically significant.

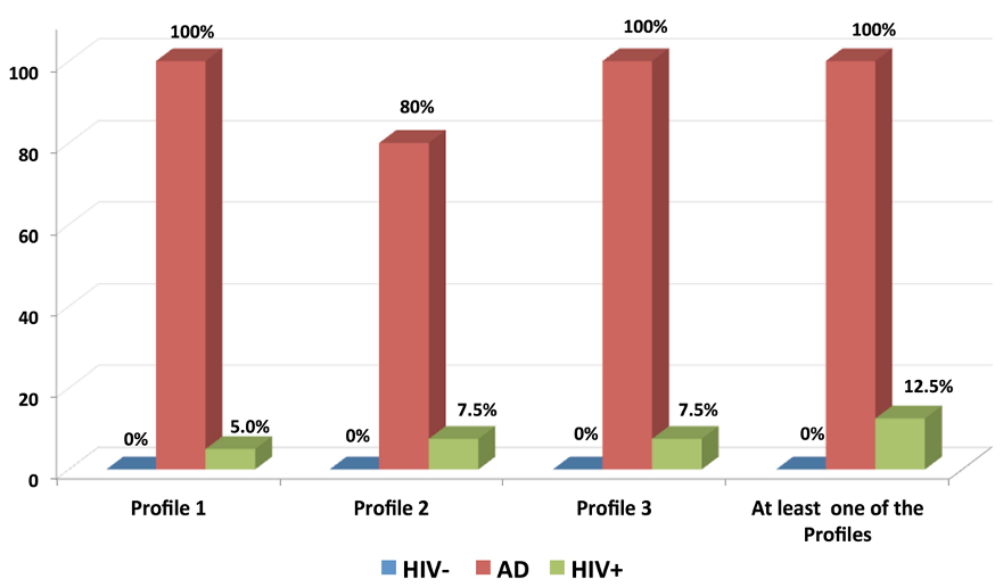

Figure 2 Prevalence of clinically relevant CSF profiles. CSF AB1-42 was missing for three cases because of insufficient CSF sample; therefore the CSF-AD like profiles were computed from 40 cases. 
Table 1 Clinical and laboratory characteristics in the entire HIV+ group

\begin{tabular}{|c|c|c|}
\hline \multirow[b]{2}{*}{ Median Nadir CD4-T+ cells/uL } & \multicolumn{2}{|c|}{ HIV+ group only } \\
\hline & 198 & IQR: 31-279 \\
\hline Median current CD4-T+ cells/ $\mu \mathrm{L}$ & 597 & IQR: $342-748$ \\
\hline $\begin{array}{l}\text { \% Plasma undetectable HIV RNA } \\
(<50 \mathrm{cp} / \mathrm{mL})^{1}\end{array}$ & $95.5 \%$ & - \\
\hline $\begin{array}{l}\text { \% CSF Undetectable HIV RNA } \\
(<50 \mathrm{cp} / \mathrm{mL})^{2}\end{array}$ & $97.6 \%$ & - \\
\hline Median HIV duration (years) & 20.5 & IQR: $14-25$ \\
\hline \% AIDS status (CDC 1993) & $70.5 \%$ & - \\
\hline$\%$ With past AIDS defining illness & $53 \%$ & - \\
\hline $\begin{array}{l}\text { Median current CART duration } \\
\text { (months) }\end{array}$ & 30 & IQR: $12-54$ \\
\hline \% MSM as HIV risk factor & $89 \%$ & - \\
\hline Average educational level (years) & 13.82 & SD: 2.95 \\
\hline$\%$ Current hAND & $61.3 \%$ & - \\
\hline$\%$ Past hAND & $31.8 \%$ & - \\
\hline \% APOE any $\varepsilon 4$ allele carriers & $30.9 \%(N=13)$ & - \\
\hline$\%$ APOE $\varepsilon 4 / \varepsilon 4$ allele carriers & $7.1 \%(\mathrm{~N}=3)$ & - \\
\hline$\%$ APOE $\varepsilon 2 / \varepsilon 3$ allele carriers & $7.1 \%(N=3)$ & - \\
\hline \% APOE $\varepsilon 3 / \varepsilon 3$ allele carriers & $61.9 \%(\mathrm{~N}=26)$ & - \\
\hline
\end{tabular}

IQR: Inter-quartile range.

SD: standard deviation.

MSM: Men who have sex with men

HAND: HIV-associated neurocognitive disorders (Frascatti 2007 diagnostic criteria).

1. Two cases had detectable plasma viral load, which were considered blips, as these cases were undetectable before and after the HIV RNA testing concomitant to the current study neuropsychological testing. Not that those cases were undetectable in the CSF.

2. One case had detectable CSF HIV RNA and this was also considered a blip for the same reasons as for plasma HIV RNA. This case was undetectable in the plasma.

Participants with past HAND status were more likely to have at least one CSF AD-like profile than those with no past HAND (31\% versus 3.7\%; $p=.03$ ). Among those with past HAND, CSF AD-like profiles were marginally more common in HAD (37.5\%) than in MND (20\%) but this was not significant as there were only 8 HAD cases, 5 MND cases and 5 individuals with a CSF AD-like profile.

In univariate analyses (Table 2) APOE genotypes incorporating $\varepsilon 4$ associated with lower levels of CSF A $\beta 1-42$ $(\mathrm{p}=.001)$. Moreover the current level of neurocognitive impairment was associated with higher $\log _{10}$ levels of $\mathrm{p}$-tau $(p=.03)$ and $\mathrm{t}$-tau $(p=.05)$.

In the multivariate model with CSF $A \beta 1-42$ as the outcome, APOE $\varepsilon 4\left(\mathrm{R}^{2}=.25 ; p=.0003\right)$ and age $\left(\mathrm{R}^{2}=.07\right.$; $p=.06)$ were optimal predictors based on the AICc criterion (model $\mathrm{R}^{2}=.32$; AICc $=536$ ). The APOE effect was driven by $\varepsilon 4 / \varepsilon 4$ cases and one $\varepsilon 3 / \varepsilon 4$ (Figure 3 represents the univariate correlation for clarity). The unusual positive and weak effect of age on CSF A 1-42 suggests a survivor bias in our cohort. This effect represents a
Table 2 Correlations between individual CSF biomarkers, AD risk markers, HIV/HAND risk markers, current overall neurocognitive impairment and past HAND

\begin{tabular}{|c|c|c|c|}
\hline \multicolumn{2}{|c|}{ Correlations between } & \multirow{2}{*}{$\begin{array}{l}r \\
-0.09\end{array}$} & \multirow{2}{*}{$\begin{array}{l}p \\
0.59\end{array}$} \\
\hline$\overline{A P O E}$ & $\log _{10}$ CSF t-tau & & \\
\hline Age & & 0.16 & 0.31 \\
\hline Nadir CD4 & & -0.19 & 0.22 \\
\hline HIV duration & & 0.04 & 0.78 \\
\hline Past HAND & & 0.10 & 0.50 \\
\hline GDS (current) & & 0.29 & 0.05 \\
\hline APOE & $\log _{10}$ CSF p-tau & -0.11 & 0.47 \\
\hline Age & & 0.08 & 0.61 \\
\hline Nadir CD4 & & -0.18 & 0.26 \\
\hline HIV duration & & -0.05 & 0.73 \\
\hline Past HAND & & 0.15 & 0.33 \\
\hline GDS (current) & & 0.32 & 0.03 \\
\hline APOE & CSF A $A 1-42$ & -0.50 & 0.001 \\
\hline Age & & 0.16 & 0.31 \\
\hline Nadir CD4 & & -0.09 & 0.58 \\
\hline HIV duration & & -0.11 & 0.51 \\
\hline Past HAND & & -0.06 & 0.70 \\
\hline GDS (current) & & 0.18 & 0.26 \\
\hline $\log _{10}$ CSF p-tau & $\log _{10}$ CSF t-tau & 0.96 & $<.0001$ \\
\hline CSF AB1-42 & $\log _{10}$ CSF t-tau & 0.48 & 0.002 \\
\hline CSF AB1-42 & $\log _{10}$ CSF p-tau & 0.53 & 0.0004 \\
\hline
\end{tabular}

APOE Genotypes were coded as follows: no $\varepsilon 4=1$; heterozygotes $\varepsilon 4 / \varepsilon 2$ or $\varepsilon 4 /$ $\varepsilon 3=2$; genotypes: homozygotes $\varepsilon 4 / \varepsilon 4=3$. Note that the correlation for APOE is driven by the $\varepsilon 4 / \varepsilon 4$ cases and one $\varepsilon 4 / \varepsilon 3$ case.

We used Pearson and point-biserial correlations as appropriate.

The Global Deficit Score (GDS) is a summary score that is an average of all the deficit scores across the test battery, and it grades normal vs. impaired performance between 0-5. A higher GDS indicated greater current overall impairment.

Past HAND: History of HAND yes was coded 1 ; no was coded 0.

non-significant small correlation in univariate analyses (see Table 2), but impacted the multivariate model.

In the first multivariate model with CSF $\log _{10} \mathrm{p}$-tau as the outcome, and using all relevant study predictors, the AICc selected the $\operatorname{GDS}(p=.13)$ as the optimal predictor and the overall model was weak (model $\mathrm{R}^{2}=.05$; AICc $=12$ ) as correlations between predictors affected the direct explanatory power of the GDS. For example; the GDS was associated with past HAND $(p=.0008)$ so the increase in p-tau reflects both current and past HAND (see Additional file 3). In the second run of the model, APOE and past HAND were excluded a priori, the GDS $(p=.03)$ was selected by the AICc. However the overall model was only slightly improved, so the combination of factors still have a relatively weak explanatory power (model $\mathrm{R}^{2}=.10$; AICc $=11.5)$. To highlight the effect of the GDS, we present the univariate correlation in Figure 4. 


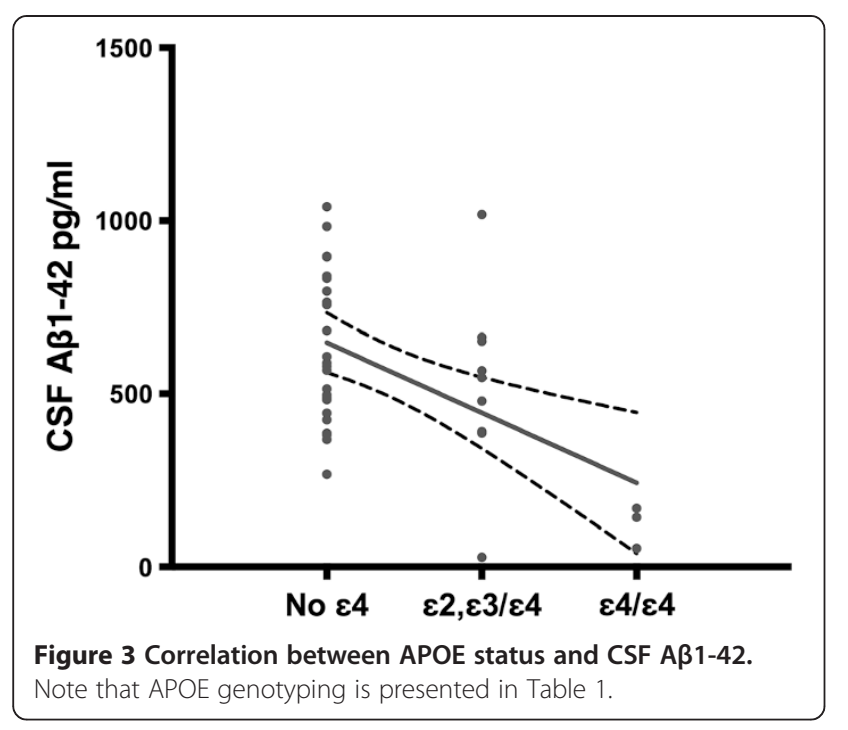

\section{Discussion}

The study is the first to associate CSF AD-like profiles with current HAD, past MND and past HAD. While the number of cases with a CSF AD-like profile is small, this may be explained by poor survival over the years before cART was

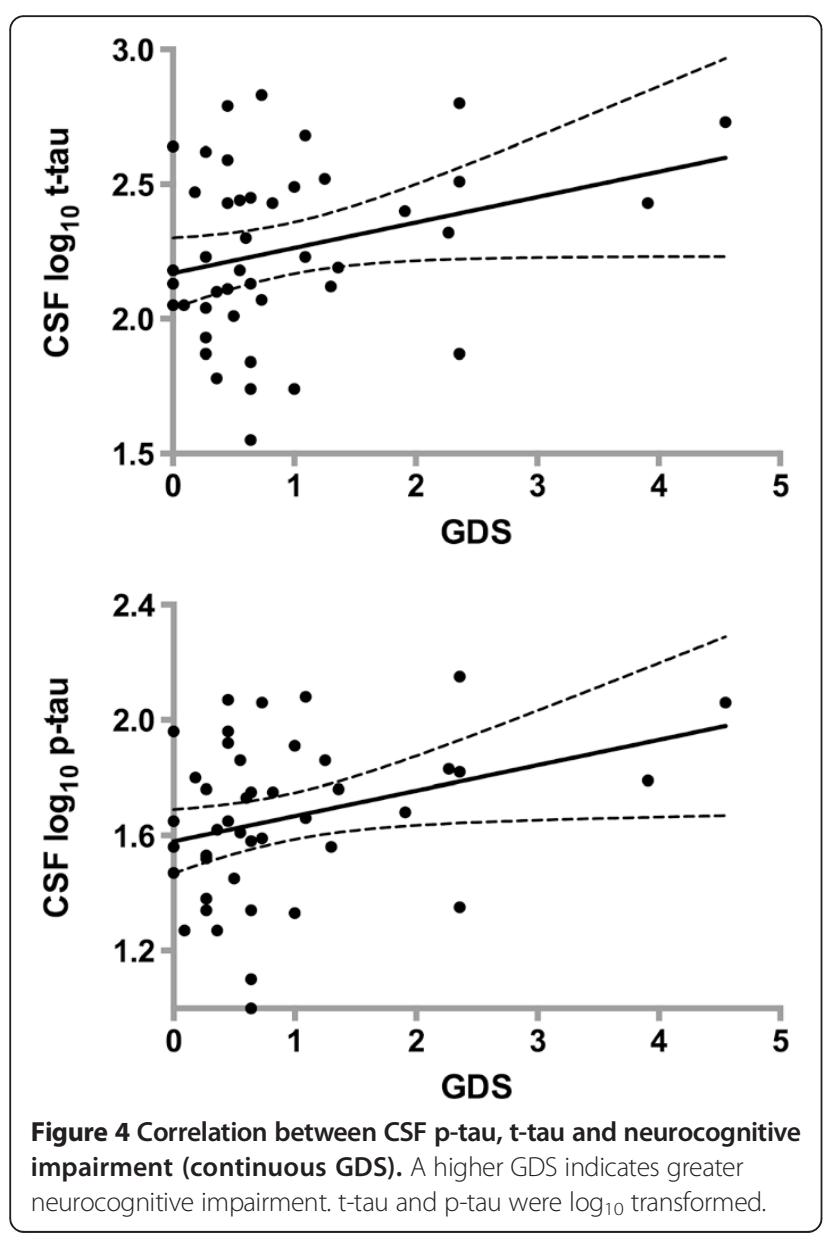

optimal. Despite this, it appears that HAD is either a risk for low CSF AD-like profile or the reverse - which would predict increases in HAD as the $\mathrm{HIV}+$ population ages. Because our study is cross-sectional, it is unclear how HIV-related brain injury may have affected levels of CSF biomarkers, though this would be consistent with the impact of past HAND.

This study is the first to include both measurements of CSF neurodegenerative markers and APOE profiling in stable optimally treated HIV+ individuals. A novel finding was that $\mathrm{APOE} \varepsilon 4$ moderated CSF A $\beta 1-42$ levels. In subjects without HIV, APOE $\varepsilon 4$ carriage is associated with lower CSF A $\beta 1-42$ concentrations [34]. This correlation emerges in subjects aged 46-65 years (the average age of our cohort) and is clearer in those aged 66-89 years. Similarly, HIV- homozygous carriers of the APOE 4 allele have lower CSF A $\beta 1-42$ levels than heterozygote carriers [34]. Although our sample had a low APOE $\varepsilon 4 / \varepsilon 4$ prevalence [cf: median $11.6 \%$; range $(4.9 \%-18.3) \%$ in the Australian population:] [35], the effect was robust and maintained in multivariate analyses. As APOE genotype is a significant risk factor for $\mathrm{AD}$ [7] and affects postmortem beta-amyloid burden in HAND [36], longitudinal studies are warranted.

Our other main finding was that neurocognitive impairment was associated with higher CSF p-tau and t-tau levels. This is not seen in normal aging. For example cognitively healthy individuals do not have elevated levels of p-tau and t-tau, but $\sim 19 \%$ show abnormal CSF A $\beta 1-42$ concentrations [34,37]. This could mean that p-tau and $\mathrm{t}$-tau mark a greater risk for current HAND in middle-aged $\mathrm{HIV}+$ individuals [38].

Lastly, past immune compromise and longer HIV duration may increase AD risk, as both are risk factors for HAND [39]. This was not evident here as the $n$ value was low.

Our findings yield useful information for longitudinal study addressing neurodegeneration in stably treated HIV infection, and to in persons aged over $50-60$ years. APOE genotyping and CSF biomarkers should be investigated as individual continuous outcomes as well as AD-like cut offs. This strategy will help determine the clinical relevance of a CSF neurodegenerative marker panel.

The inclusion of older participants at this stage of the HIV epidemic has some caveats as most are survivors of the pre-cART era. Thus our data may contain some survivor bias. Therefore until cohorts with no survivor bias (i.e.,: individuals started on cART) are available, the neuroHIV community should remain cautious of a lack of detection or weak signal of accelerated neurodegeneration in aging $\mathrm{HIV}+$ persons. Such cohorts will display a lower prevalence of HAD but MND is still relatively common in persons on cART $[17,20]$ and ANI can predict neurocognitive deterioration [40]. 
In addition CSF biomarkers and APOE genotype, other mechanisms might explain $\beta$-amyloid dysregulation in chronic HIV infection. Indeed our data suggests that higher CSF A $\beta 1-42$ concentrations may correlate with CSF p-tau and t-tau levels. This is counter-intuitive as lower CSF A $\beta 1-42$ and increased tau mark AD. However the inverse relationship was only seen in a sub-set of participants. In others CSF A $\beta 1-42$ concentrations are normal or slightly elevated (Figure 1). Elevation of CSF A $\beta 1-42$ concentrations may represent an anti-inflammatory effect [41]. Supporting this interpretation is the fact that CSF neopterin correlated with CSF A $\beta 1-42(\mathrm{r}=.40 ; \mathrm{p}=.009)$.

\section{Conclusions}

At first glance, our results seem contrary to studies which found no evidence of amyloid burden in HIV+ individuals [42] or no relationship between HAND and APOE $\varepsilon 4$ genotype [12]. However we show that APOE $\varepsilon 4$ genotype moderates the expression of CSF A $\beta 1-42$ in participants with HAND. Moreover as in larger studies [29], we find that APOE does not directly impact HAND. Perhaps as most individuals are still relatively young ( $<60$ years), amyloid build up is incomplete and tau pathologies may be a more common trigger for brain damage. With evidence that the CSF biomarker risk for $\mathrm{AD}$ in the current $\mathrm{HIV}+$ sample is over 10 times greater (12.5\%) than in the Australian population of the same age [43], this shows the need for a longitudinal study of the aging HIV+ population.

\section{Limitations}

Our study offers new insights into the CSF biomarker pathologies in HIV infection, but the design is crosssectional so the clinical consequences are unclear. Our study included a medium size sample, but the participants were well characterized and uniform in having achieved viral suppression on cART. This excludes HIV replication as a cause of HAND compared to previous studies [4-6]. Some APOE $\varepsilon 4$ homozygotes may have died before cART was available (i.e., survival bias), as the prevalence of APOE $\varepsilon 4 / \varepsilon 4$ is lower than in the Australian general population.

\section{Additional files}

Additional file 1: Correspondence between the 1988 ADC and the 2007 American Academy of Neurology HAND diagnosAc nomenclature.

Additional file 2: Table S1. Study groups' demographic characteristics. Additional file 3: Table S2. Correlations between multivariate analyses predictors.

\section{Abbreviations}

AD: Alzheimer's disease; APOE: Apolipoprotein E; CART: combined antiretroviral therapy; CSF: Cerebrospinal fluid; ELISA: Enzyme-linked immunoassay; GDS: Global deficit score; HAD: HIV-associated dementia; HAND: HIV-associated neurocognitive disorder; HIV: Human immunodeficiency virus; MND: Mild neurocognitive disorder; t-tau: total tau; p-tau: phosphorylated tau.

\section{Competing interests}

Lucette A Cysique received honoraria from Abbvie Ltd, CogState Ltd, ViiV healthcare, partial salary support in 2012 from Mercks Sharp Dome and CogState Ltd. Dr. Cysique is funded by a National Health and Medical Research Council of Australia Career Development Fellowship \#APP1045400 and has research support from Abbvie Ltd, ViiV healthcare and the Australian National Association of People Living with HIV/AIDS (NAPWA).

Timothy Hewitt: the author declares that they have no competing interests. Julianna Croitoru-Lamoury: the author declares that they have no competing interests.

Kevin Taddei: the author declares that they have no competing interests. Ralph N Martins: the author declares that they have no competing interests. Constance SN Chew: the author declares that they have no competing interests. Nicholas WS Davies: the author declares that they have no competing interests. Patricia Price: the author declares that they have no competing interests. Bruce J Brew received payment as board member for GlaxoSmithKline, Biogen Idec, ViiV Healthcare, Merck Serono,; has received grants from National Institutes of Health USA, National Health and Medical Research Council of Australia and from MSRA; received honoraria from ViiV Healthcare, Boehringer Ingelheim, Abbott, Abbvie, and Biogen Idec; receives royalties from HIV Neurology (Oxford University Press, 2001) and Palliative Neurology (Cambridge University Press, 2006); received travel/ accommodation expenses covered or reimbursed by Abbott; and has research support funding from El Lilly, GlaxoSmithKline, ViiV Healthcare, Merck Serono, and St. Vincent's Clinic Research Foundation.

\section{Authors' contributions}

LC was the study primary investigator, designed the study, collected some of the neuropsychological data, conducted and interpreted the statistical analyses and led the manuscript writing. TH participated in the assays analyses and interpretation as well as in the manuscript writing. JCL conducted the biological assays, supervised the assays interpretation and analyses as well as participated in the manuscript writing. KT and RM were responsible for the collection of some of the CSF data in HIV- and AD cases, and participated in the overall data interpretation and manuscript writing. CC conducted the genetic analyses, participated in genetic analyses interpretation. ND collected the CSF data in HIV+ cases, participated in overall data interpretation and manuscript writing. PP supervised the genetic analyses and interpretation, participated in overall data interpretation and manuscript writing. BB oversaw the overall study conduct as the primary site investigator, facilitated the CSF data collection, analyses and storage, oversaw and participated in overall data analyses and interpretation as well as manuscript writing. All authors read and approved the final manuscript.

\section{Author details}

'University of New South Wales, St. Vincent's Hospital Clinical School, Sydney, Australia. ${ }^{2}$ Neuroscience Research Australia, Sydney, Australia. ${ }^{3}$ Department of Neurology St. Vincent's Hospital, Sydney, Australia. ${ }^{4}$ St. Vincent's Centre for Applied Medical Research, Sydney, Australia. ${ }^{5}$ University of Notre Dame, Sydney, Australia. ${ }^{6}$ Centre of Excellence for Alzheimer's disease Research \& Care, School of Medical Sciences, Edith Cowan University, Mount Lawley, Australia. ${ }^{7}$ Sir James McCusker Alzheimer's Disease Research Unit, Hollywood Private Hospital, Nedlands, Australia. ${ }^{8}$ School of Psychiatry and Clinical Neurosciences, University of Western Australia, Perth, Australia. ${ }^{9}$ School of Biomedical Science, Curtin University of Technology, Bentley, Australia.

${ }^{10}$ Department of Neurology, Chelsea \& Westminster Hospital, London, UK.

Received: 14 March 2014 Accepted: 11 March 2015

Published online: 01 April 2015

\section{References}

1. Krut JJ, Zetterberg H, Blennow K, Cinque P, Hagberg L, Price RW, et al. Cerebrospinal fluid Alzheimer's biomarker profiles in CNS infections. J Neurol. 2013;260(2):620-6.

2. Mattsson N, Zetterberg H, Hansson O, Andreasen N, Parnetti L, Jonsson M, et al. CSF biomarkers and incipient Alzheimer disease in patients with mild cognitive impairment. JAMA. 2009;302(4):385-93. doi:10.1001/jama.2009.1064. 
3. Cysique LA, Moffat K, Moore DM, Lane TA, Davies NW, Carr A, et al. HIV, vascular and aging injuries in the brain of clinically stable HIV-infected adults: a (1)H MRS study. PLoS One. 2013;8(4):e61738.

4. Brew BJ, Pemberton L, Blennow K, Wallin A, Hagberg L. CSF amyloid beta42 and tau levels correlate with AIDS dementia complex. Neurology. 2005;65(9):1490-2. doi:10.1212/01.wnl.0000183293.95787.b7 [doi].

5. Gisslen M, Krut J, Andreasson U, Blennow K, Cinque P, Brew BJ, et al. Amyloid and tau cerebrospinal fluid biomarkers in HIV infection. BMC Neurol. 2009;9:63. doi:10.1186/1471-2377-9-63 [doi].

6. Clifford DB, Fagan AM, Holtzman DM, Morris JC, Teshome M, Shah AR, et al. CSF biomarkers of Alzheimer disease in HIV-associated neurologic disease. Neurology. 2009;73(23):1982-7. doi:10.1212/WNL.0b013e3181c5b445 [doi].

7. Bertram L, Lange C, Mullin K, Parkinson M, Hsiao M, Hogan MF, et al. Genome-wide association analysis reveals putative Alzheimer's disease susceptibility loci in addition to APOE. Am J Hum Genet. 2008;83(5):623-32. doi:10.1016/j.ajhg.2008.10.008 [doi].

8. Farrer LA, Cupples L, Haines JL, Hyman B, Kukull WA, Mayeux R et al. Effects of age, sex, and ethnicity on the association between apolipoprotein e genotype and alzheimer disease: A meta-analysis. JAMA. 1997;278(16):1349-56. doi:10.1001/jama.1997.03550160069041.

9. Wisniewski T, Frangione B, Apolipoprotein E. A pathological chaperone protein in patients with cerebral and systemic amyloid. Neurosci Lett. 1992;135(2):235-8. doi:http://dx.doi.org/10.1016/0304-3940(92)90444-C.

10. Valcour V, Shikuma C, Shiramizu B, Watters M, Poff P, Selnes OA, et al. Age, apolipoprotein E4, and the risk of HIV dementia: the Hawaii Aging with HIV Cohort. J Neuroimmunol. 2004;157(1-2):197-202.

11. Burt TD, Agan BK, Marconi VC, He W, Kulkarni H, Mold JE, et al. Apolipoprotein (apo) E4 enhances HIV-1 cell entry in vitro, and the APOE epsilon4/epsilon4 genotype accelerates HIV disease progression. Proc Natl Acad Sci U S A. 2008;105(25):8718-23.

12. Morgan EE, Woods SP, Letendre SL, Franklin DR, Bloss C, Goate A, et al. Apolipoprotein E4 genotype does not increase risk of HIV-associated neurocognitive disorders. J Neurovirol. 2013;19(2):150-6.

13. Dunlop O, Goplen AK, Liestol K, Myrvang B, Rootwelt H, Christophersen B, et al. HIV dementia and apolipoprotein E. Acta Neurol Scand. 1997;95(5):315-8.

14. Joska JA, Combrinck M, Valcour VG, Hoare J, Leisegang F, Mahne AC, et al. Association between apolipoprotein E4 genotype and human immunodeficiency virus-associated dementia in younger adults starting antiretroviral therapy in South Africa. J Neurovirol. 2010;16(5):377-83.

15. Pemberton LA, Stone E, Price P, van Bockxmeer F, Brew BJ. The relationship between ApoE, TNFA, IL1a, IL1b and IL12b genes and HIV-1-associated dementia. HIV Med. 2008;9(8):677-80.

16. Lane TA, Moore DM, Batchelor J, Brew BJ, Cysique LA. Facial emotional processing in HIV infection: Relation to neurocognitive and neuropsychiatric status. Neuropsychology. 2012;26(6):713-22. doi:10.1037/a0029964 [doi].

17. Cysique LA, Heaton RK, Kamminga J, Lane T, Gates TM, Moore DM, et al. HIV-associated neurocognitive disorder in Australia: a case of a high-functioning and optimally treated cohort and implications for international neuroHIV research. J Neurovirol. 2014;20(3):258-68.

18. Carey CL, Woods SP, Gonzalez R, Conover E, Marcotte TD, Grant I, et al. Predictive validity of global deficit scores in detecting neuropsychological impairment in HIV infection. J Clin Exp Neuropsychol. 2004;26(3):307-19.

19. Heaton RK, Miller SW, Taylor MJ, Grant I. Revised comprehensive norms for an expanded Halstead-Reitan Battery: Demographically adjusted neuropsychological norms for African American and Caucasian adults Scoring Program. Odessa: FL: Psychological Assessment Ressources; 2004.

20. Blackstone K, Moore DJ, Franklin DR, Clifford DB, Collier AC, Marra CM, et al. Defining neurocognitive impairment in HIV: deficit scores versus clinical ratings. Clin Neuropsychol. 2012;26(6):894-908.

21. Heaton RK, Marcotte TD, Mindt MR, Sadek J, Moore DJ, Bentley H, et al. The impact of HIV-associated neuropsychological impairment on everyday functioning. J Int Neuropsychol Soc. 2004;10(3):317-31. doi:10.1017/ S1355617704102130.

22. Chelune G, Heaton R, Lehman R. Neuropsychological and personality correlates of patient's complaints of disability. In: Tarter R, Goldstein G, editors. Advances in clinical neuropsychology. New York: New York Plenum Press; 1986. p. 95-126.

23. Cysique LA, Bain MP, Lane TA, Brew BJ. Management issues in HIV-associated neurocognitive disorders. Neurobehavioral HIV Med. 2012;4(1):63-73.

24. Price RW, Brew BJ. The AIDS dementia complex. J Infect Dis. 1988;158(5):1079-83.
25. Antinori A, Arendt G, Becker JT, Brew BJ, Byrd DA, Cherner M, et al. Updated research nosology for HIV-associated neurocognitive disorders. Neurology. 2007;69(18):1789-99. doi:10.1212/01.WNL.0000287431.88658.8b.

26. Mattsson N, Andreasson U, Persson S, Arai H, Batish SD, Bernardini S, et al. The Alzheimer's association external quality control program for cerebrospinal fluid biomarkers lessons from multicenter studies on CSF biomarkers for Alzheimer's disease. Alzheimers Dement. 2011;7(4):386-95 e6.

27. McKhann G, Drachman D, Folstein M, Katzman R, Price D, Stadlan EM. Clinical diagnosis of Alzheimer's disease: report of the NINCDS-ADRDA Work Group under the auspices of Department of Health and Human Services Task Force on Alzheimer's Disease. Neurology. 1984;34(7):939-44.

28. American Psychiatric Association. Diagnostic and Statistical Manual of Mental Disorders, Text Revision (DSM-IV-TR). Washington, DC: APA; 2000.

29. Hansson O, Zetterberg H, Buchhave P, Londos E, Blennow K, Minthon L. Association between CSF biomarkers and incipient Alzheimer's disease in patients with mild cognitive impairment: a follow-up study. Lancet Neurol. 2006;5(3):228-34. doi:10.1016/S1474-4422(06)70355-6 [doi].

30. Diniz BS, Pinto Junior JA, Forlenza OV. Do CSF total tau, phosphorylated tau, and beta-amyloid 42 help to predict progression of mild cognitive impairment to Alzheimer's disease? A systematic review and meta-analysis of the literature. World J Biol Psychiatry. 2008:9(3):172-82. doi:10.1080/15622970701535502 [doi].

31. Caselli RJ, Reiman EM. Characterizing the Preclinical Stages of Alzheimer's Disease and the Prospect of Presymptomatic Intervention. J Alzheimers Dis. 2012. doi:10.3233/JAD-2012-129026 [doi].

32. Mothobi NZ, Brew BJ. Neurocognitive dysfunction in the highly active antiretroviral therapy era. Curr Opin Infect Dis. 2012;25(1):4-9.

33. Burnham KP, Anderson DR. Multimodel inference: understanding AIC and BIC in model selection. Sociological Methods Res. 2004;33(2):261-304. doi: 10.1177/0049124104268644.

34. Popp J, Lewczuk P, Frommann I, Kolsch H, Kornhuber J, Maier W, et al. Cerebrospinal fluid markers for Alzheimer's disease over the lifespan: effects of age and the APOEepsilon4 genotype. J Alzheimers Dis. 2010;22(2):459-68. doi:10.3233/JAD-2010-100561 [doi].

35. Ward A, Crean S, Mercaldi CJ, Collins JM, Boyd D, Cook MN, et al. Prevalence of apolipoprotein E4 genotype and homozygotes (APOE e4/4) among patients diagnosed with Alzheimer's disease: a systematic review and metaanalysis. Neuroepidemiology. 2012;38(1):1-17. doi:10.1159/000334607 [doi].

36. Soontornniyomkij V, Moore DJ, Gouaux B, Soontornniyomkij B, Tatro ET, Umlauf A et al. Cerebral beta-amyloid deposition predicts HIV-associated neurocognitive disorders in APOE epsilon4 carriers. AIDS. 2012. doi:10.1097/QAD.0b013e32835a117c [doi]

37. Paternico D, Galluzzi S, Drago V, Bocchio-Chiavetto L, Zanardini R, Pedrini L, et al. Cerebrospinal fluid markers for Alzheimer's disease in a cognitively healthy cohort of young and old adults. Alzheimers Dement. 2012:8(6):520-7. doi:10.1016/j.jalz.2011.10.003 [doi].

38. Steinbrink F, Evers S, Buerke B, Young P, Arendt G, Koutsilieri E et al. Cognitive impairment in HIV infection is associated with MRI and CSF pattern of neurodegeneration. Eur J Neurol. 2012. doi:10.1111/ene.12006 [doi].

39. Brew BJ. Evidence for a change in AIDS dementia complex in the era of highly active antiretroviral therapy and the possibility of new forms of AIDS dementia complex. AIDS. 2004;18 suppl 1:S75-8.

40. Grant I, Franklin Jr DR, Deutsch R, Woods SP, Vaida F, Ellis RJ, et al. Asymptomatic HIV-associated neurocognitive impairment increases risk for symptomatic decline. Neurology. 2014;82(23):2055-62.

41. Soscia SJ, Kirby JE, Washicosky KJ, Tucker SM, Ingelsson M, Hyman B, et al. The Alzheimer's disease-associated amyloid beta-protein is an antimicrobial peptide. PLoS One. 2010;5(3):e9505.

42. Ances BM, Benzinger TL, Christensen JJ, Thomas J, Venkat R, Teshome M, et al. 11C-PiB imaging of human immunodeficiency virus-associated neurocognitive disorder. Arch Neurol. 2012;69(1):72-7.

43. Cysique LA, Bain MP, Brew BJ, Murray JM. The burden of HIV-associated neurocognitive impairment in Australia and its estimates for the future. Sex Health. 2011;8(4):541-50. 